\title{
Urethral stricture disease: Measuring success in treatment
}

\author{
Timothy 0. Davies, MD, FRCSC
}

Assistant Professor, Division of Urology, Master University, Juravinski Hospital, Hamilton, ON

See related article on page 260 .

Cite as: Can Urol Assoc J 2012;6(4):265. http://dx.doi.org/10.5489/cuaj.12221

U rethral stricture disease and its management are complex. The UREThRAL stricture score (USS) as described by Wiegand and Brandes is a novel method to describe and quantify urethral stricture disease. ${ }^{1}$

To develop the USS, they chose factors they believed to be important and assigned a point value to each domain. The appealing UREThRAL acronym was used to recall the domains of etiology, number of strictures, luminal obliteration, location and length. Retrospectively they analyzed a group of postoperative urethral reconstructive patients to see if the score correlated with a subjective surgical complexity score. As they point out, there would be some debate as to the value of the surgical complexity score. Excision and primary anastomosis is doubtlessly the simplest of the open urethral reconstructive techniques and most would agree that combined graft and flap tissue transfer is used for the most complex stricture disease. Variability in surgical training and surgeon preference would be a significant confounder to the treatment complexity score. The value of a quantifiable urethral stricture score would be in comparing the scores to patient outcomes.

Outcome measures continue to be one of the major hurdles to overcome in providing good quality research in reconstructive urology. Measurement of patient outcomes following urethral reconstructive surgery is not standardized. Many different methods have been used in the past to evaluate "success" following urethroplasty. Cystoscopy, urethral x-ray studies, uroflow and post-void residuals have been used to capture outcomes. Assessing the quality of life - the most important outcome - has yet to be standardized. Investigators have used non-validated questionnaires (like the AUA symptom score). Recently, Jackson and colleagues have taken a first step to develop a stricture specific health related quality of life questionnaire. $^{2}$ The current challenge facing reconstructive urology is to develop a validated and standardized method of assessing patients pre- and postoperatively.
In the development of The UREThRAL stricture score, the authors have delineated the important factors in determining the complexity of a stricture. This is valuable reminder to all urologists. At the initial evaluation of stricture disease, the factors pointed out by the authors (etiology, number of strictures, luminal obliteration, location and length) are the keys to determine the severity of the stricture itself. The length, as the authors point out, is heavily weighted and it is accepted to be the most likely determinant of both outcome and treatment. ${ }^{3,4}$ Longer and more complex strictures should signal the urologist to consider early open surgical intervention rather than pursuing futile and repeated endoscopic management (urethrotomy/dilatation). The poor outcomes ${ }^{5}$ and cost ineffectiveness ${ }^{6}$ of repeated endoscopic treatment of urethral strictures are well-described in the literature. Failed endoscopic treatment may be a useful additional factor in the USS. This may encourage earlier consideration for urethral reconstruction, which would benefit the patient and the health care system.

Competing interests: None declared.

\section{References}

1. Wiegand LR, Brandes SB. The UREThRAL stricture score: A novel method for describing anterior urethral strictures. Can Urol Assoc J 2012;6:260-4. http://dx.doi.org/10.5489/cuaj.12048

2. Jackson MJ, Sciberras J, Mangera A, et al. Defining a patient-reported outcome measure for urethral stricture surgery. Eur Urol 2011;60:60-8. htrp://dx.doi.org/10.1016/i.eururo.2011.03.003

3. Albers P, Fichtner J, Bruhl P. Long-term results of internal urethrotomy. J Urol 1996;156:161 1-4.

4. Morey AF, Lin HC, DeRosa CA, et al. Fossa navicularis reconstruction: impact of stricture length on outcomes and assessment of extended meatotomy (first stage Johanson) maneuver. J Urol 2007;177:184-7, discussion 187.

5. Steenkamp J, Heyns C, de Kock ML. Internal urethrotomy versus dilation as treatment for male urethral strictures: a prospective, randomized comparison. J Urol 1997;157:98-101.

6. Rourke K, Jordan G. Primary urethral reconstruction: The cost minimized approach to the bulbous urethral stricture. J Urol 2005;173:1206-10. http://dx.doi.org/10.1097/01.ju.0000154971.05286.81

Correspondence: Dr. Timothy 0. Davies, Assistant Professor, Division of Urology, McMaster University, Juravinski Hospital, 711 Concession St, Section B3, Hamilton, ON L8V 1C3; fax: 905381-7037; tdavies@mcmaster.ca 